\title{
Working with primary care clinicians and patients to introduce strategies for increasing referrals for pulmonary rehabilitation
}

\author{
Faye Foster ${ }^{1}$, Rosie Piggott ${ }^{2}$, Lucy Riley ${ }^{3}$ and Roger Beech ${ }^{4}$ \\ ${ }^{1}$ Former Research Associate, Health Services Research, Health Service Research Unit, Keele University, Keele, \\ Staffordshire, UK \\ ${ }^{2}$ Clinical GP Research Fellow, Research Institute of Primary Care and Health Sciences, Keele University, Keele, \\ Staffordshire, UK \\ ${ }^{3}$ Research Associate, Biostatistics, Health Service Research Unit, Keele University, Keele, Staffordshire, UK \\ ${ }^{4}$ Reader in Health Services Research, Research Institute of Primary Care and Health Sciences, Keele University, Keele, \\ Staffordshire, UK
}

Background: Pulmonary rehabilitation (PR), a programme of exercise, education and psycho-social support, is recommended for patients with chronic obstructive pulmonary disease but referral rates are relatively low compared with need. Aim: Working with primary care clinicians (GPs and practice nurses) from eight practices, this project developed strategies for influencing clinician and patient behaviours as a means of increasing referral rates for PR. Methods: A participatory action research design was employed. Semi-structured questionnaires captured clinicians' baseline knowledge of PR and their ideas for increasing referrals. Actionable changes were then recommended. Audits (at baseline, mid-point and end of project) were used to assess and initiate improvements in the quality of practice data about PR referrals. The impacts of these changes were explored via further clinician surveys (free text questionnaire). Semi-structured questionnaires, posted to patients eligible for PR, assessed their characteristics, and, where applicable, their views on PR referral processes and reasons for not wanting PR. Findings: The baseline survey of clinicians $(n=22)$ revealed inadequate knowledge about PR, particularly among GPs. Actionable changes recommended included in-house education sessions, changes to practice protocols, and 'pop-ups' and memory aids (mugs and coasters) to prompt clinician/patient discussions about PR. Audit findings resulted in changes to improve the quality and availability of coded information about patients eligible for PR. These changes, supported by clinicians ( $n=9)$ in the follow-up survey, aimed to facilitate and increase the quality of patient/ clinician discussions about PR. Findings from the patient survey ( $n=126$, response rate $25.7 \%$ ) indicate that such changes will increase the uptake of PR as patients who accepted a referral for PR provided more positive feedback about their discussions with clinicians. Conclusions: The strategies introduced were relatively easy to implement and the anticipated advantage is more patients accessing the health and quality of life benefits that PR offers.

Key words: behaviour; COPD; delivery of health care; participatory research; pulmonary rehabilitation

Received 19 December 2014; revised 19 March 2015; accepted 19 April 2015 ;

first published online 15 June 2015

Correspondence to: Dr Roger Beech, Research Institute of Primary Care and Health Sciences, Keele University, Keele, Staffordshire ST5 5BG, UK. Email: r.beech@keele.ac.uk 


\section{Introduction}

Chronic obstructive pulmonary disease (COPD) is a chronic condition which causes severe breathlessness, a persistent cough, an increased likelihood of anxiety and depression and a reduced quality of life (Pooler and Beech, 2014). Internationally, it is a leading cause of morbidity and mortality and in England COPD is the second leading cause of unplanned hospital admissions [Department of Health, 2012; The Global Initiative for Chronic Obstructive Lung Disease (GOLD), 2013].

Pulmonary rehabilitation (PR) is a supervised programme of exercise training, education and psycho-social support for people who are functionally disabled by COPD [usually defined as a score of 3 or above on the Medical Research Council (MRC) dyspnoea scale]. For participating patients, PR improves exercise tolerance and health-related quality of life, and reduces fatigue, dyspnoea, anxiety and depression (Coventry and Lacasse et al., 2006; Hind, 2007). PR also reduces the direct costs of COPD by decreasing patients' unplanned use of health care resources and in particular their unplanned hospital admissions (Griffiths et al., 2001; Cecins et al., 2008). As a result, national and international guidelines recommend PR for managing patients with moderate to severe COPD [National Institute for Health and Care Excellence (NICE), 2011; GOLD, 2013].

However, patient uptake of PR remains low relative to need. A systematic search of surveys and audits by Johnston and Grimmer-Somers (2010) found that only $3-16 \%$ of eligible patients were referred for PR. A systematic review by Keating et al. (2011) found that the referring doctor is influential in patients' willingness to accept an offer of PR, with patients being less likely to accept the referral if they do not know the doctor or if the doctor seems unclear about the benefits of PR for patients. Johnston et al. (2013) surveyed Australian GPs to obtain their perspectives on the causes of low referral rates for PR. Key factors were GPs being unclear about the nature of PR, not knowing how to refer patients and being unsure about the benefits of PR for the patients under their care.

Patient attitudes are also a factor with the low uptake of PR among eligible patients being linked to them: not regarding PR as beneficial, being unable to accommodate PR attendance within their existing schedule of daily activities, finding attendance difficult because of the timing or location of PR sessions (Keating et al., 2011). These findings were re-iterated in a study by Moore et al. (2012) and a systematic review by Thorpe et al. (2014) which also found that patients were concerned that they would be unable to meet the physical demands of the PR programme. It has also been found that current smokers and people who live alone or who are widowed or divorced are less likely to accept the offer of a referral for PR (Keating et al., 2011; Moore et al., 2012).

Hence, both clinician and patient attitudes and behaviours are a cause of low referral rates for PR. By drawing on the knowledge of patients, primary care staff (GPs and practice nurses), and academics from Keele University, the project described in this developmental paper aimed to generate and introduce strategies for influencing staff and patient behaviour as a means of increasing referral rates for PR.

In addition to published guidelines and research findings, a number of local factors acted as stimuli for the study. The setting for the project was Stoke-on-Trent, an area with high levels of COPD (Health and Social Care Information Centre, 2014). Some staff from participating practices had recently attended a COPD professional development course hosted by Keele University. Here they heard a talk by a lecturer in physiotherapy who presented the evidence base to support PR. This was followed by an insightful and emotive talk given by members of Stoke and North Staffordshire's Breathe Easy Group (individuals with COPD and their informal carers) who described the benefits that they had gained from PR. These talks prompted one of the primary care teams in attendance to undertake an audit of data held within their COPD register. The results (which were shared with colleagues on the course) reflected current concerns about the low use of PR by eligible patients. The practice had 168 patients eligible for PR of whom only $16(9.5 \%)$ had been referred to and/or completed PR. Of the remaining eligible patients, $87(51.8 \%)$ had declined PR and $65(38.7 \%)$ had no documented record to indicate that a member of the primary care team had spoken to them about PR. 


\section{Study aims and methods}

\section{Aims}

Using a mixed method and participatory action research design (Bergold and Thomas, 2012), this project aimed to develop and introduce strategies to influence the behaviour of both primary care clinicians (GPs and practice nurses) and patients with COPD as a means of increasing referrals for PR. For clinicians, the focus was on making them more aware of the benefits of PR and on ensuring that they identified and offered PR to all eligible patients. For patients with COPD, the aim was to help them to make a more informed choice about whether to accept a referral for PR.

Nine primary care practices from a single locality of Stoke CCG were invited to participate in the project. These practices were targeted because they have some of the largest COPD registers in Stoke, ranging from around 160 to 400 patients per practice. From a pragmatic perspective, this recruitment strategy also allowed the Keele team to build on the links that they had formed with staff from these practices who had attended the professional development course referred to above. To recruit practices, F.F. and R.P. initially met with the Locality Chair and Business Manager to explain the project who then arranged access for the team to present details of the project at practice meetings. Out of nine practices in the locality, eight agreed to join the project. To facilitate the delivery of the project, each practice was asked to identify a 'liaison person' (usually the practice manager) who acted as the main point of contact between the practice and the Keele team. The project ran from April 2013 to June 2014.

\section{Methods: influencing clinician behaviour}

A semi-structured questionnaire was used to assess GPs' and practice nurses' baseline (June/ July 2013) knowledge of and attitudes to PR. Copies of this questionnaire were given to the liaison person in each practice who then identified those clinicians who were involved in the care of patients with COPD and asked them to complete the questionnaire.

The baseline questionnaire also asked respondents for their ideas on how referrals to PR might be increased. Based upon the feedback and a review of relevant literature, a briefing note was then issued to practices in November 2013. This briefing note included 'actionable' suggestions for standardising knowledge of PR among GPs and nurses and for increasing referral rates to PR. In May 2014 the project team contacted practices again to obtain feedback on which actionable suggestions had been adopted and how these had affected referral procedures for PR. A self-completed open text survey was the preferred method of obtaining feedback but telephone conversations with key staff were also used. As in the baseline survey, the practice liaison person was responsible for distributing the survey instrument to relevant clinicians and encouraging them to complete it.

Audits of practice data were also undertaken at three time points across the project: June 2013, December 2013 and May 2014. The audits had two roles: to assess the ease with which practices were able to provide information about patients who were eligible for PR (information that can inform clinician/patient discussions) and to monitor the impacts of the project over time. To support the audits, each practice was offered the use of an experienced administrator to conduct the database searches: one practice accepted this offer.

For the June and December audits, the following information was requested from practices:

a. COPD register size.

b. Number of patients eligible for PR.

c. Number of eligible patients with a PR code (indicating that a documented conversation about PR has taken place between a clinician and a patient).

For the final audit in May 2014, the information requested was extended to include:

d. The coded outcome of clinician/patient conversations about $P R$

- Referred for PR;

- PR referral declined;

- PR programme completed;

- Did not complete PR programme.

The additional information about the outcome of clinician/patient discussions was not requested in the initial audits because discussions with practice staff indicated that it was unlikely to be available. However, it was requested in the final audit to assess if the project had influenced the scope and quality of coding about PR. 


\section{Methods: investigating patient behaviour}

In each practice, the practice liaison person was asked to provide a list giving the contact details of patients eligible for PR (MRC dyspnoea scale score 3 or above). They were also asked to subdivide patients on the list into those with and without a code for PR. During visits to practices, an evaluator (F.F.) sent to each patient on the lists a letter written on behalf of the practice that included: a project information sheet, a survey questionnaire and a stamped addressed envelope (for the anonymous return of the questionnaire to the evaluation team. These mail shots took place during January 2014 and during this task the patient lists never left participating practices.

The survey questionnaires were designed by the study team and covered issues that past research has shown affects the uptake of PR by patients (Keating et al., 2011; Moore et al., 2012; Thorpe et al., 2014). There were two variations of the questionnaire. The one sent to patients with a code for PR initially gathered information on their characteristics (see Table 1 for a complete list of the data gathered). Using a Likert scale, patients were then asked to respond to a series of questions about PR referral processes (see Figures 4-6 for details of the questions posed and types of response options). Finally, patients were asked if they had accepted a referral for PR and if not their reasons for declining (free text answer).

The questionnaire sent to patients without a PR code also gathered information about their characteristics followed by a question to confirm that a clinician had never spoken to them about PR. If they indicated that, in fact, such a conversation had taken place they were then invited to answer the additional questions about their views on referral processes and their decisions regarding a referral for PR.

\section{Data analysis and ethical approval}

Feedback from the staff questionnaires and telephone interviews were grouped, key themes identified and responses from GPs and nurses compared (where relevant). Descriptive statistics were used to analyse the data collected by the audits to show the availability of the information requested and trends over the time. Feedback from the patient questionnaires was analysed using descriptive statistics and responses compared between those who accepted/declined a referral for PR. Additional analysis of patient characteristics compared those who were spoken to/not spoken to about PR.

Details of the project were sent to the NRES Committee North West - Liverpool Central.

Table 1 Characteristics of patient sample (126 patients)

\begin{tabular}{|c|c|c|c|c|}
\hline Patient characteristic & $\begin{array}{l}\text { Has discussed PR with } \\
\text { a clinician } \\
84(66.7 \%)\end{array}$ & $\begin{array}{l}\text { Has not discussed PR } \\
\text { with a clinician } \\
42(33.3 \%)\end{array}$ & $\begin{array}{l}\text { Accepted a } \\
\text { referral for PR } \\
59(100.0 \%)\end{array}$ & $\begin{array}{l}\text { Declined a } \\
\text { referral for PR } \\
20(100.0 \%)\end{array}$ \\
\hline \multicolumn{5}{|l|}{ Marital status } \\
\hline Married & $46(54.8 \%)$ & $17(40.5 \%)$ & $37(62.7 \%)$ & $9(45.0 \%)$ \\
\hline Widowed & $18(21.4 \%)$ & $13(31.0 \%)$ & $12(20.3 \%)$ & $4(20.0 \%)$ \\
\hline Divorced & $12(14.3 \%)$ & $9(21.4 \%)$ & $4(6.8 \%)$ & $5(25.0 \%)$ \\
\hline Separated & $3(3.6 \%)$ & $0(0 \%)$ & $1(1.7 \%)$ & $2(10.0 \%)$ \\
\hline Never married & $5(6.0 \%)$ & $3(7.1 \%)$ & $5(8.5 \%)$ & $0(0 \%)$ \\
\hline \multicolumn{5}{|l|}{ Living status } \\
\hline Lives alone & $31(36.9 \%)$ & $20(47.6 \%)$ & $19(32.2 \%)$ & $9(45.0 \%)$ \\
\hline Lives with family/friend & $52(61.9 \%)$ & $22(52.4 \%)$ & $39(66.1 \%)$ & $11(55.5 \%)$ \\
\hline $\begin{array}{l}\text { Lives in residential/nursing } \\
\text { home }\end{array}$ & $1(1.2 \%)$ & $0(0 \%)$ & $1(1.7 \%)$ & $0(0 \%)$ \\
\hline Current smoker & $19(22.6 \%)$ & $8(19.0 \%)$ & $8(13.6 \%)$ & $9(45.0 \%)$ \\
\hline $\begin{array}{l}\text { Currently prescribed oxygen } \\
\text { therapy }\end{array}$ & $13(15.5 \%)$ & $2(4.8 \%)$ & $12(20.3 \%)$ & $1(5.0 \%)$ \\
\hline $\begin{array}{l}\text { Has more than one chronic } \\
\text { medical condition }\end{array}$ & $41(48.8 \%)$ & $26(61.9 \%)$ & $32(54.2 \%)$ & $6(30.0 \%)$ \\
\hline
\end{tabular}

$\mathrm{PR}=$ pulmonary rehabilitation . 
They indicated that the project was a service evaluation and therefore did not require NHS REC approval.

\section{Results}

\section{Influencing staff behaviour}

\section{Baseline situation}

In total, 22 questionnaires for assessing baseline knowledge of and attitudes to PR were returned by clinicians from the eight participating practices (9 from GPs and 13 from practice nurses). Findings indicated a lack of knowledge among GPs about what PR involves, with nurses having a greater awareness of the nature of PR and its role. In addition GPs were less cognisant than nurses about patient eligibility for PR. All practice nurses surveyed indicated correctly that patients with an MRC dyspnoea scale score of 3 and above are eligible for PR whereas GPs' responses were less succinct. Survey findings also revealed a lack of clear within-practice guidelines about whose responsibility it was to make referrals for PR and a lack of clarity about referral processes.

\section{Actionable suggestions}

Feedback from clinicians in the baseline survey indicated that further efforts were needed to increase their awareness of the nature and merits of PR and help them to identify eligible patients and initiate the referral process. As a consequence, the following actionable suggestions were included in a briefing note issued to practices in November 2013:

- Thirty minute in-house training and awareness events (particularly for GPs) run by PR service representatives focusing on opportunistic discussion of PR with patients, the referral process and the nature of local services.

- Integration of clear prompts for referral on COPD review templates and consideration to be given to prompts for discussion and referral for use by GPs during routine appointments with COPD patients and appointments for exacerbations of COPD.

- Creation of practice protocols with clear practice-specific referral strategies about who refers and when.

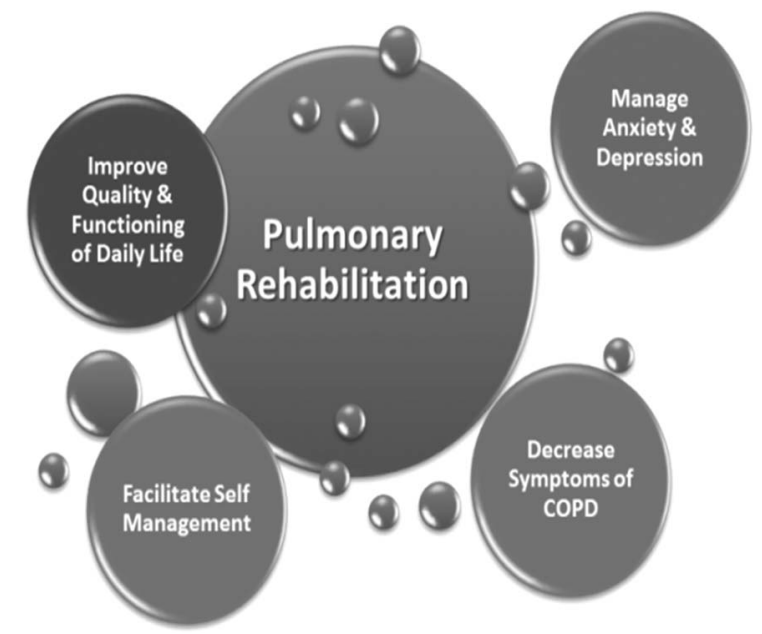

Figure 1 Logo used as PR reminder. PR = pulmonary rehabilitation; COPD $=$ Chronic Obstructive Pulmonary Disease

- The use of memory aids for GPs and nurses in the form of a recognisable logo (Figure 1) printed on mugs and coasters and distributed to clinicians in each participating practice.

Feedback on the uptake and usefulness of these suggestions was provided by nine clinicians (five nurses and four GPs) and the liaison person at each practice on behalf of the practice teams.

Six practices requested the 30 -min training and awareness events with staff regarding them as an effective and flexible way of passing on knowledge about PR. Holding the events at a time that was convenient for practice staff was seen as important. Clinicians in the two remaining practices did not feel they had a need for the events as they felt that they had already disseminated the learning obtained from their participation in the professional development course referred to previously.

The addition of a prompt on the review template was seen as a simple means of reminding practice nurses to consider a referral to PR during their discussions with patients. Two practices introduced computerised 'pop-up' alerts that invited the GP to query a referral to PR for patients with an MRC dyspnoea scale score of 3 and above. GPs from these practices thought pop-up alerts were a good idea 'in theory' but repetition and erroneous suggestions for referral promoted annoyance particularly when GPs 
had to respond by closing down the pop-up before continuing the consultation.

Respondents felt that the practice protocols should primarily build upon the existing role of the practice nurse as they were regarded as being the most conversant with PR referral processes and used review appointments to make referrals for PR. GPs were seen as having a more opportunistic role in initiating PR referrals.

Finally, feedback indicated that the PR mugs and coasters were in regular use. The use of the PR logo was seen as reinforcing a positive attitude toward referral to PR.

\section{Audits of practice data}

For each practice and audit undertaken, the results presented in Figure 2 show the number of patients coded as being eligible for PR and the percentage of these with a code for PR. Six of the eight practices were able to provide data on patient eligibility for PR across each of the three time points of the audit with patient numbers by practice remaining relatively stable. Only four practices were able to provide complete information about eligible patients with a PR code and in these practices this number increased across the three time points. For the final audit, six practices were able to provide these data with results highlighting current concerns about patient access to PR as the number of eligible patients with a coded conversation about $\mathrm{PR}$ ranged from $18 \%$ to $80 \%$. Six practices were able to provide the more comprehensive information requested in the final audit (Figure 3 ) with results demonstrating that many patients decline an offer of a referral to PR when it is made.
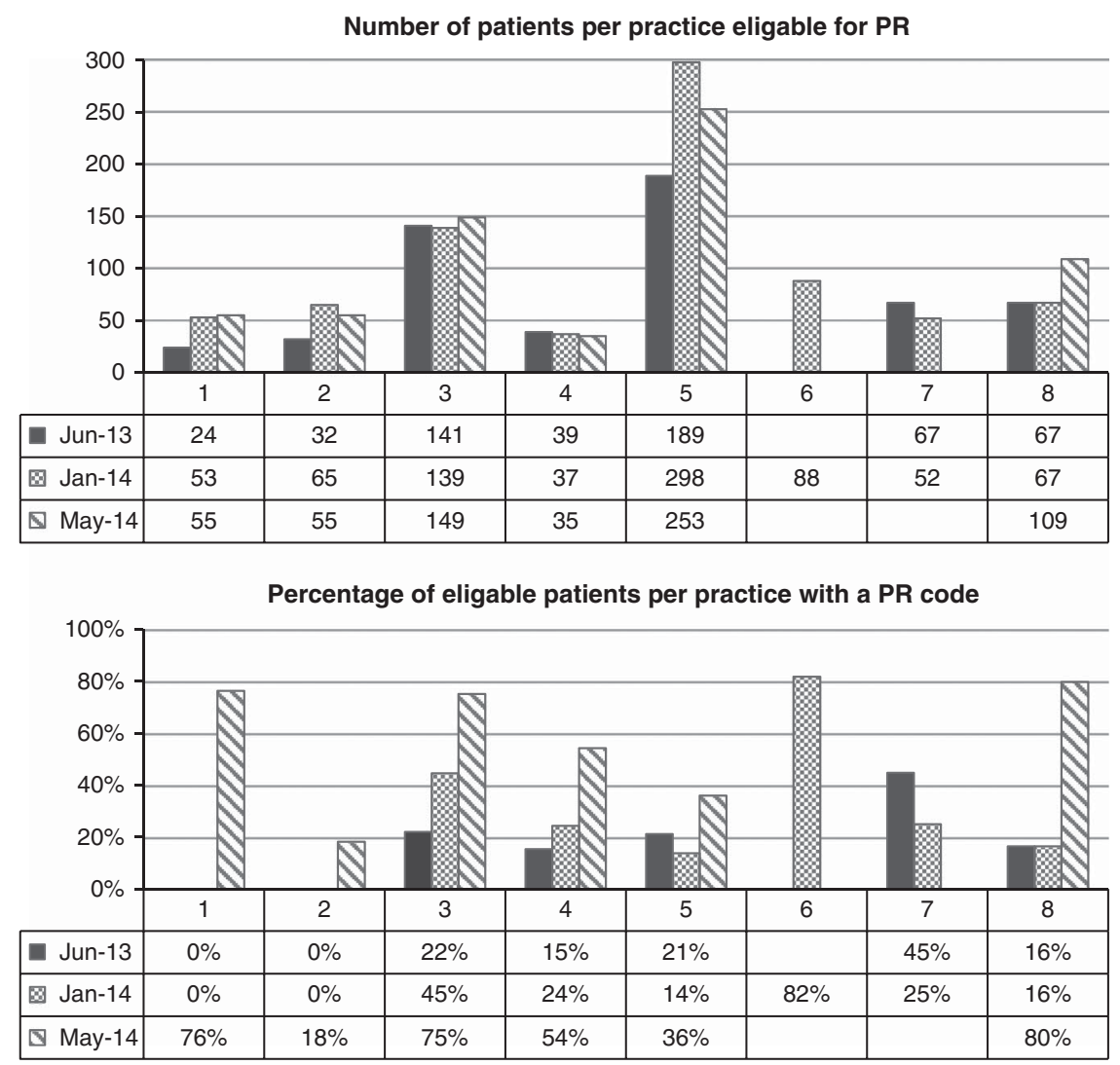

Figure 2 Eligibility and coding for PR. PR = pulmonary rehabilitation 


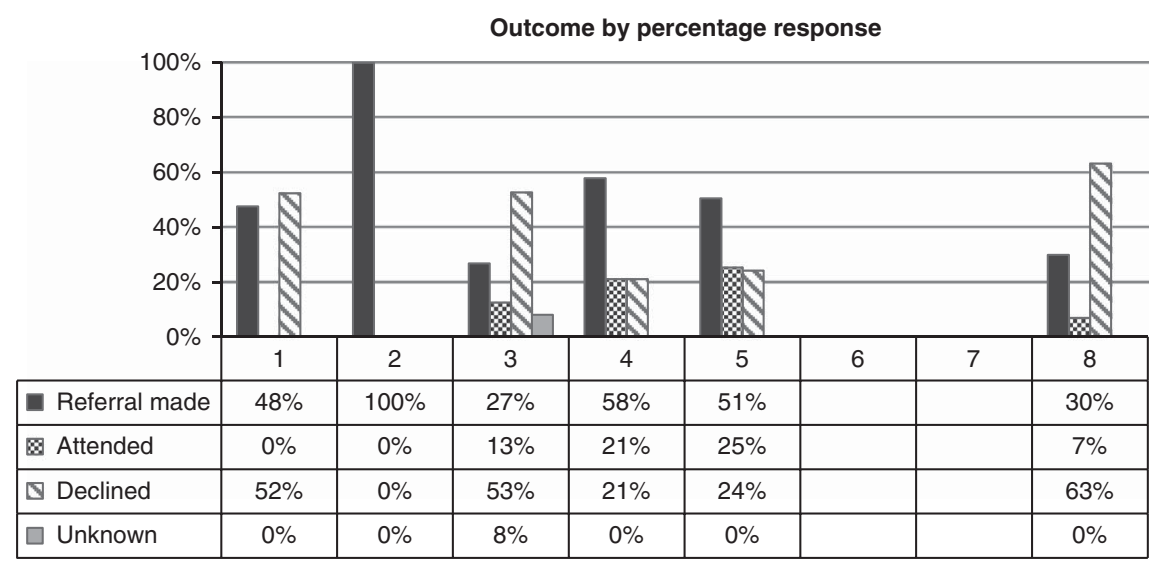

Figure 3 Outcome of patient/clinician discussions about PR by practice. PR = pulmonary rehabilitation

\section{Investigating patient behaviour}

\section{Patient sample}

In total, 490 questionnaires were posted to patients in the participating practices who were eligible for PR and 126 were returned (a response rate of $25.7 \%$ ). Of these, 59 were from patients who had a code for PR in their primary care records and 67 from those who had no code. However, the feedback from patients indicated that practice data were incomplete as 25 patients without a code for PR stated that a clinician had in fact spoken to them about PR. As a result, the returned questionnaires covered 84 patients who had discussed PR with a clinician and 42 who had not. The characteristics of these two groups of patient are compared in columns 2 and 3 of Table 1. Among those individuals who indicated that they had discussed PR with a clinician, a greater percentage were married and a lower proportion indicated that they had more than one chronic condition.

Of the 84 patients who had discussed PR with a clinician, $59(70.2 \%$ ) accepted a referral for PR, 20 $(23.9 \%)$ declined a referral and $5(5.9 \%)$ did not answer this question. Columns 4 and 5 of Table 1 compare the characteristics of the 79 patients who gave a definite response to this question. Among those who accepted a referral for PR a low percentage were current smokers. Current smokers were more evident among those who declined a referral but still the majority in this group indicated that they did not smoke. The group of individuals who accepted a referral also included a higher percentage of individuals who were on oxygen therapy or had more than one chronic condition. Finally, among those who declined a referral for PR, a greater proportion lived alone and were divorced or separated.

Nine of the 20 individuals who declined a referral gave reasons for their decision. Health issues were raised by three patients: two felt that they were not well enough to attend PR and one preferred to remain at home during cold weather. Three more felt that they did not need a referral at this stage while the remaining three faced logistical difficulties in accessing PR (transport issues or inability to get time off work).

Remaining results focus on the characteristics of the consultation process and further explore differences between the 59 individuals who accepted a referral for PR and the 20 who did not. Nurses, closely followed by doctors were by far the most common source of information about PR for both patient groups with doctors and nurses having similar success rates in terms of getting patients to accept a referral for PR. Patients who accepted a referral were more likely to feel that they were given the correct amount of information about PR in an understandable way during their discussions with clinicians (Figure 4). Likewise, these patients were more likely to feel that their questions and anxieties about PR had been addressed (Figure 5). This was reflected in those who accepted a referral having a more positive view of the referral process (Figure 6). 
Qu: "Were you given the right amount of information to help you choose?"

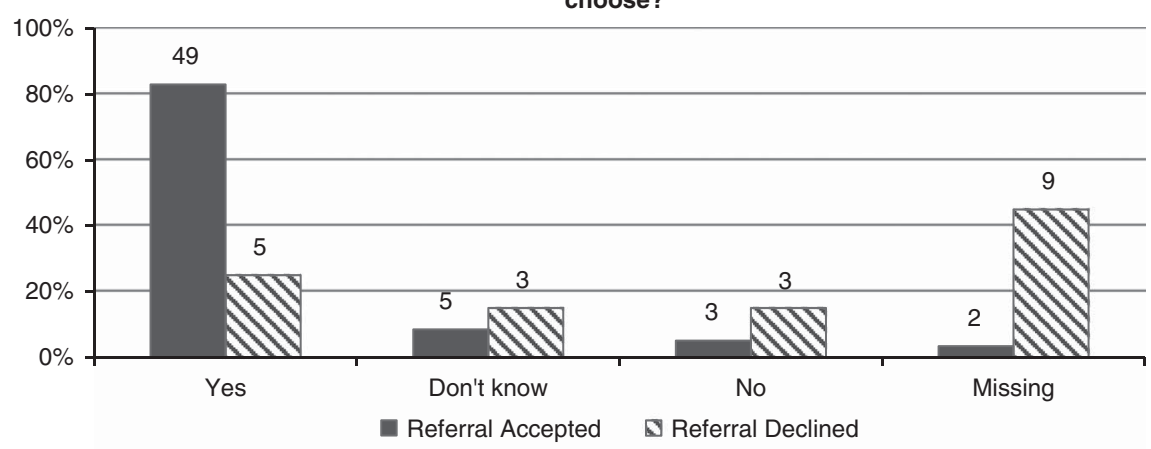

Qu: "Did a HCP explain the purpose of PR in a way you could

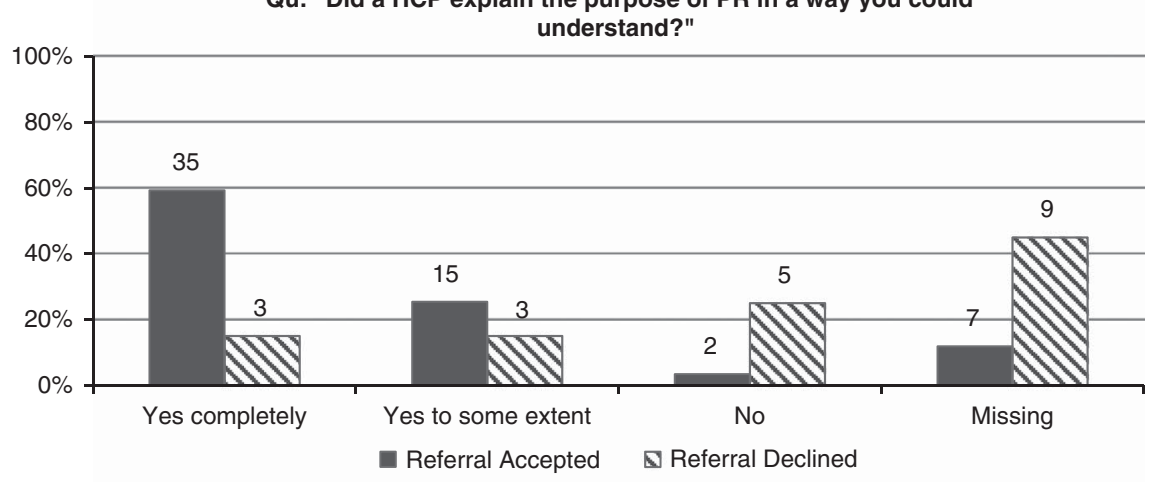

Qu: "On reflection, did you get the information about PR that mattered to you?"

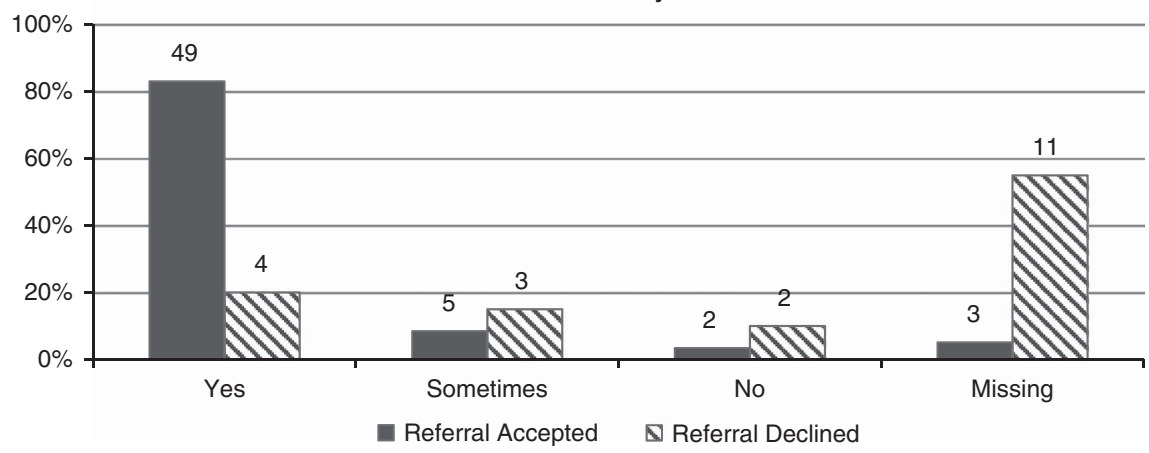

Figure 4 Feedback on the quality of information provided about PR (79 patients). PR = pulmonary rehabilitation; $\mathrm{HCP}=$ healthcare professional

\section{Discussion}

PR can improve the health and quality of life of patients and reduce their unplanned demands for health services (NICE, 2011; GOLD, 2013). However, referral rates are low relative to need due to both clinician and patient related factors (Keating et al., 2011; Moore et al., 2012; Thorpe et al., 2014; Johnston et al., 2013). The developmental project described here aimed to provide an increased understanding of factors that were affecting patient access to PR as a means of Primary Health Care Research \& Development 2016; 17: 226-237 
Qu: "When you had an important question to ask, did you get answers that you could understand?"

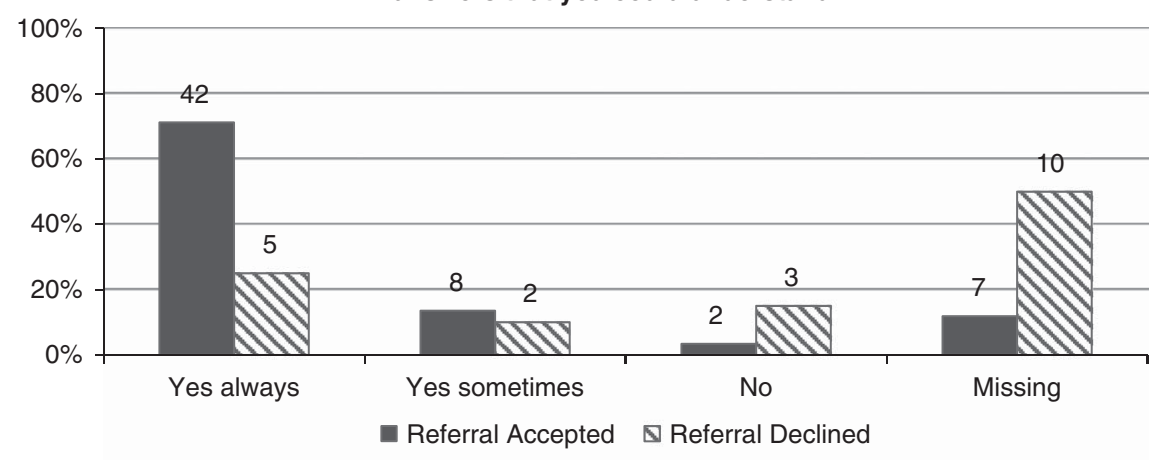

Qu: "If you had any fears or anxieties about PR did a HCP discuss them with you?"

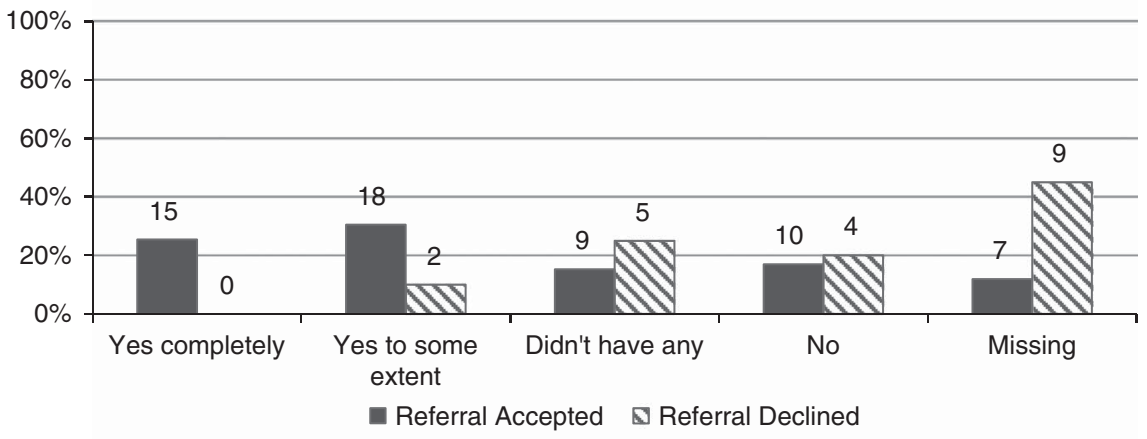

Qu: "How confident are you that the HCP who referred you had the knowledge of what PR involved?"

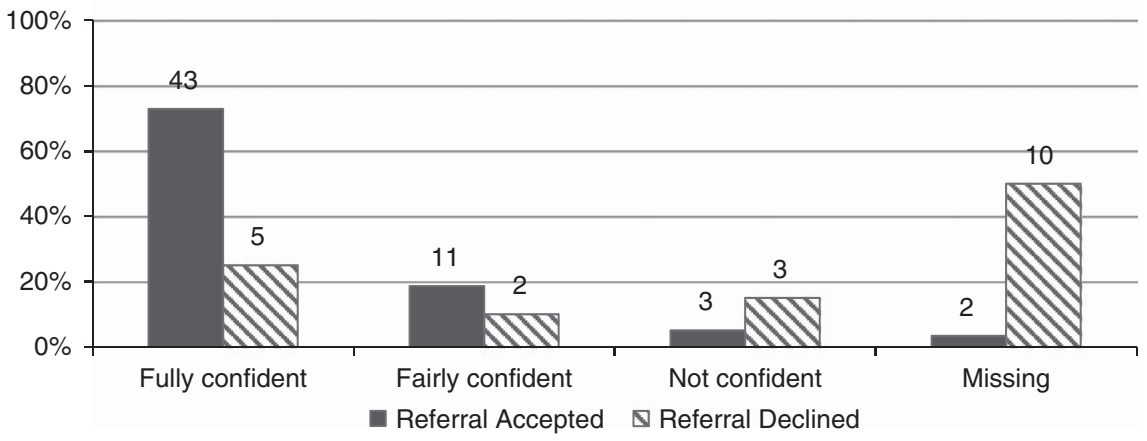

Figure 5 Feedback on the extent to which patient concerns about PR were addressed (79 patients). PR = pulmonary rehabilitation; $\mathrm{HCP}=$ healthcare professional

generating local strategies to increase referral rates. This discussion of the project's findings focus on three issues that affect referral rates: clinicians being able to describe the nature and benefits of PR; individuals being willing to accept referrals for Primary Health Care Research \& Development 2016; 17: 226-237
PR when they are offered; patient data held within practices being of sufficient quality to inform patient/clinician discussions about PR.

Patients not recognising the benefits of PR is a known barrier to referral (Keating et al., 2011), 
Qu: "Overall, how satisfied are you with the referral service you have received from your HCP?"

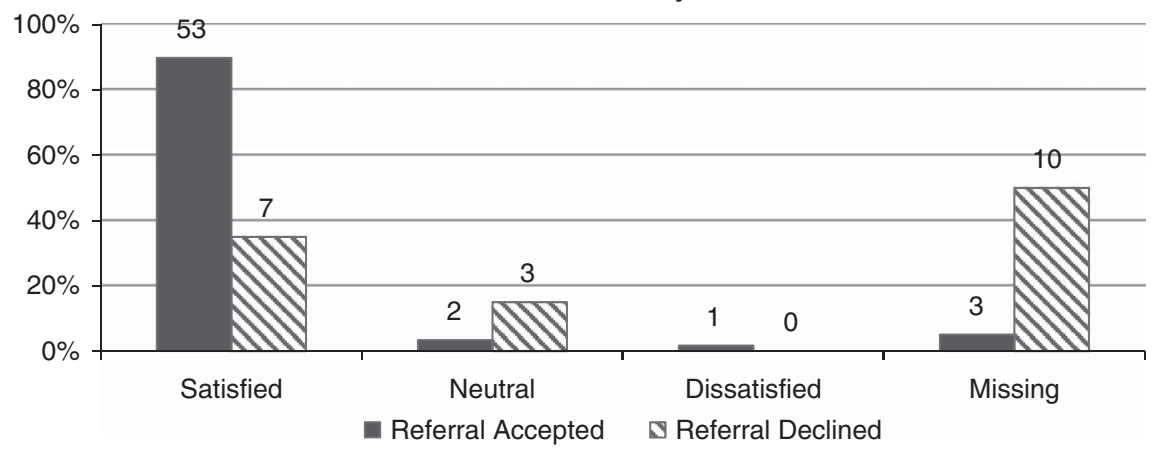

Figure 6 Overall satisfaction with the referral process (79 patients). HCP $=$ healthcare professional

and was given as a reason for declining PR by some respondents to the patient survey. Clinicians will find it difficult to address this negative view if they themselves are unclear about the benefits of this service: at baseline, GPs in particular reported a lack of knowledge and understanding about PR. To address this deficiency, this project introduced in-house training and awareness events [a tactic suggested in research elsewhere (Johnston et al., 2011)] and feedback indicates that these proved beneficial. The quality of clinician/patient discussions could also be enhanced by a greater use of resources that describe PR such as leaflets, DVDs and web links: feedback from the patient survey indicated that these are rarely used at present.

Findings from the patient survey [and those from previous studies (Harris et al., 2008a; Johnston et al., 2013)] support a hypothesis that improving the quality of the patient/clinician conversation can increase referral rates for PR. Patients who accepted a referral for PR gave more positive feedback about their discussions with clinicians. For example, they reported having important questions answered in a way they understood, having their anxieties satisfactorily dealt with, and being given enough information by knowledgeable clinicians. However, where patients' questions were not answered, or answered unsatisfactorily, they were more likely to decline a referral for PR.

Survey findings also exposed characteristics that appear to make individuals more reluctant to accept a referral for PR: in particular, being a current smoker and living alone. Free text responses also demonstrated that health and logistical concerns can cause patients to reject a referral for PR. Such findings are in keeping with previous research (Harris et al., 2008b; Keating et al., 2011; Moore et al., 2012; Thorpe et al., 2014). Ways of persuading such 'hard to reach' groups could be a focus of further training and awareness events as survey findings demonstrated that it is possible to encourage individuals with these characteristics to accept a referral for PR (e.g., 32.2\% of those who accepted a referral lived alone). Additional training could also be used to address clinician perceptions about characteristics that make patients less likely to want a referral for PR as survey findings suggested that clinicians were less likely to discuss PR with patients who lived alone and who had more than one chronic condition. In practice, patients with more than one chronic condition were in the majority among those who accepted a referral for PR.

Clearly, a pre-requisite for any patient/clinician conversation about PR is clinicians being aware of individuals who are eligible for this intervention. Patient data held within practices is a potential source of such information and most practices were able to extract this information across each of the time points of the audit. The extraction of information to indicate if such patients had previously discussed PR with a clinician proved more problematic although for the final audit six of the eight practices were able to provide this information. Findings (and those from the patient survey) re-affirmed concerns about patient access 
to $\mathrm{PR}$, with the percentage of eligible patients with a PR code ranging from $18 \%$ to $80 \%$. For the final audit, data on the outcome of patient/clinician discussions about PR were also requested (referral made, attended, declined). Most practices were able to provide this information, demonstrating that the audits introduced by this study were helping to improve the quality of practice data about PR.

The omission of coding for PR in eligible patients' records does not necessarily mean that they have not had a conversation with a clinician about PR. However, inadequate coding does limit the ability of data held within practices to support patient/clinician discussions. The changes introduced as part of this study help to ensure that clinicians are aware of those patients who are eligible for PR, if they have been spoken to about PR in the past and, if so, the outcome of those conversations. Even if the proportion of patients accepting or declining a referral for PR remains unchanged, simply ensuring that more eligible patients have a discussion with a clinician about PR will lead to an overall increase in patient access to PR.

The project introduced other strategies to remind clinicians to discuss PR with patients: mugs and coasters containing a PR logo, prompts within the protocols used by nurses and prompts for GPs during their consultations with patients. Feedback indicated that each of these approaches had its merits with prompts within nurse templates being seen as the most influential.

Did the strategies that were introduced result in an increase in referral rates for PR from participating practices? In six of the eight practices, the results presented in Figure 2 indicate that across the three time points of the audit there was an increase in the percentage of eligible patients with a coded conversation about PR and that many of these conversations led to a referral for PR (Figure 3). In practice though, any rise in referral rates is likely to be gradual as clinicians use their review appointments throughout the year to engage with patients who have not previously been offered PR.

Strengths of this developmental study are that it addressed an issue that local clinicians had identified as important. The study's participatory approach also meant that the strategies that were developed for tackling this problem were informed by feedback from local clinicians and individuals with COPD. The weaknesses of the study mainly surround the level and quality of information obtained from patients.

Findings from the patient survey support those found elsewhere but individuals who declined a referral for PR were probably under-represented among respondents and many of the 'declined attendance' group did not answer all questions posed. This led to a high number of missing values which means the comparisons of the characteristics and attitudes of individuals who accepted or rejected a referral for PR need to be viewed with caution. The relatively small number of subjects within categories also meant that statistical tests were not undertaken as any findings observed were likely to be by chance. The use of a free text response for capturing patients' reasons for declining a referral for PR had some success but a semi-structured style of questioning may have resulted in more detailed feedback. Finally, time and resource constraints meant that the original aim of seeking patients' views on ways of increasing referral rates for PR was not possible. Patientproposed strategies generated in research elsewhere include: individuals who have completed PR acting as mentors for newly referred patients (Moore et al., 2012) and clinician/patient discussions focusing on 'real life' benefits derived from PR such as individuals being more able to play with grandchildren (Harris et al., 2008b).

The scope and representativeness of the feedback obtained from clinicians in participating practices is a further potential area of weakness. A liaison person in each practice coordinated the distribution of the survey questionnaires as they were seen as being best placed to encourage clinicians to give priority to completing study questionnaires. At baseline 22 clinicians from across the eight practices completed the survey, although it is unclear what proportion these staff represented of the total number of clinicians who provided care for patients with COPD. For the follow-up survey, only nine clinicians (plus the liaison person at each practice) provided feedback. However, without the help of the liaison person, this figure is likely to have been lower.

In summary, clinician and patient attitudes and behaviours are a cause of low referral rates for PR. Measures to increase clinicians' knowledge about PR and to alert them to patients that are eligible 
for this intervention, can be used to influence clinician behaviour. Clinicians will then be better placed to influence patient behaviour because they will be more able to describe the benefits of PR and to discuss any concerns that patients may have about this intervention. The types of strategy introduced in this study were each relatively easy to implement and the anticipated pay-off is an increase in the number of patients accessing PR and receiving the health and quality of life benefits that it delivers.

\section{Acknowledgements}

Stoke CCG provided the funds for this study. Staff and patients from the practices who participated in this study are also thanked for their support.

\section{References}

Bergold, J. and Thomas, S. 2012: Participatory research methods: a methodological approach in motion [110 paragraphs]. Forum Qualitative Sozialforschung/Forum: Qualitative Social Research, [S.I], v. 13, n. 1, jan. 2012. ISSN 1438-5627. Retrieved 15 December 2014 from http://www.qualitativeresearch.net/index.php/fqs/article/view/1801/3334

Cecins, N., Geelhoed, E. and Jenkins, S.C. 2008: Reduction in hospitalization following pulmonary rehabilitation in patients with COPD. Australian Health Review 32, 415-22.

Coventry, P.A. and Hind, D. 2007: Comprehensive pulmonary rehabilitation for anxiety and depression in adults with chronic obstructive pulmonary disease: systematic review and meta-analysis. Journal of Psychosomatic Research 63, 551-65.

Department of Health. 2012: An outcomes strategy for COPD and asthma: NHS companion document. Retrieved 31 October 2014 from https://www.gov.uk/government/uploads/system/ uploads/attachment_data/file/216531/dh_134001.pdf.

The Global Initiative for Chronic Obstructive Lung Disease (GOLD). 2013: Global strategy for diagnosis, management and prevention of COPD. Updated February 2013. Retrieved 31 October 2014 from www.goldcopd. org/uploads/ users/files/GOLD_Report_2013_Feb20.pdf

Griffiths, T.L., Phillips, C.J. and Davies, S. 2001: Cost effectiveness of an outpatient pulmonary rehabilitation program. Thorax 56, 779-84.
Harris, D., Hayter, M. and Allender, S. 2008a: Factors affecting the offer of pulmonary rehabilitation to patients with chronic obstructive pulmonary disease by primary care professionals: a qualitative study. Primary Health Care Research \& Development 8, 280-90.

Harris, D., Hayter, M. and Allender, S. 2008b: Improving the uptake of pulmonary rehabilitation in patients with COPD. British Journal of General Practice 58, 703-10.

Health and Social Care Information Centre. 2014: Quality and Outcomes Framework (QOF) - 2013-14. Retrieved 9 December 2014 from http://www.hscic.gov.uk/article/ 2021/Website-Search?productid $=16273 \&$ q=COPD+qof $\&$ sort $=$ Relevance \&size $=10 \&$ page $=1 \&$ area $=$ both\#top

Johnston, K. and Grimmer-Somers, K. 2010: Pulmonary rehabilitation: overwhelming evidence but lost in translation. Physiotherapy Canada 62, 368-73.

Johnston, K.N., Young, M., Grimmer, K.A., Antic, R. and Frith, P.A. 2013: Barriers to, and facilitators for, referral to pulmonary rehabilitation in COPD patients from the perspective of Australian general practitioners: a qualitative study. Primary Care Respiratory Journal 22, 319-24.

Keating, A., Lee, A. and Holland, A.E. 2011: What prevents people with chronic obstructive pulmonary disease from attending pulmonary rehabilitation? A systematic review. Chronic Respiratory Disease 8, 89-99.

Lacasse, Y., Goldstein, R., Lasserson, T.J. and Martin, S. 2006: Pulmonary rehabilitation for chronic obstructive pulmonary disease. Cochrane Database of Systematic Reviews CD003793. doi: 10.1002/14651858.CD003793.pub2. Retrieved 31 October 2014 from http://onlinelibrary.wiley. com/doi/10.1002/14651858.CD003793.pub2/abstract

Moore, L., Hogg, L. and White, P. 2012: Acceptability and feasibility of pulmonary rehabilitation for COPD: a community qualitative study. Primary Care Respiratory Journal 21, 419-24.

National Institute for Health and Care Excellence (NICE). 2011: Chronic obstructive pulmonary disease quality standard. NICE quality standard 10. Retrieved 31 October 2014 from http://www.nice.org.uk/guidance/qs10/resources/guidancechronic-obstructive-pulmonary-disease-quality-standard-pdf

Pooler, A. and Beech, R. 2014: Examining the relationship between anxiety and depression and exacerbations of COPD which result in hospital admission: a systematic review. The International Journal of Chronic Obstructive Pulmonary Disease 9, 315-30. Retrieved 8 December 2014 from http:// www.ncbi.nlm.nih.gov/pmc/articles/PMC3974694/

Thorpe, O., Kumar, S. and Johnston, K. 2014: Barriers to and enablers of physical activity in patients with COPD following a hospital admission: a qualitative study. International Journal of COPD 9, 115-28. 